\title{
Use of powered mobile arm supports by people with neuromuscular conditions
}

\author{
Arun Kumar, MSc, BSc; ${ }^{1}$ Margaret Frances Phillips, MD $^{\mathbf{1 - 2 *}}$ \\ ${ }^{1}$ Division of Rehabilitation Medicine, University of Nottingham, Derby, UK; ${ }^{2}$ Derby Hospitals Foundation Trust, \\ Derby, UK
}

\begin{abstract}
This study used a mixed-methods approach with questionnaires and semistructured interviews to explore the views and experiences of people with neuromuscular conditions who use powered mobile arm supports (PMASs). All 170 users of PMASs who had neuromuscular conditions and lived within $200 \mathrm{mi}$ of the study center were contacted by post. Of the 22 who expressed an interest, 13, aged 13 to $69 \mathrm{yr}$, took part. Participants had been using the PMAS from 6 mo to $8 \mathrm{yr}$, and the majority had Duchenne muscular dystrophy. Interviews took place in participants' homes or by telephone, and participants completed the ABILHAND and the Upper-Limb Functional Index and provided details regarding their diagnosis and experience with the PMAS. Themes that emerged were the positive influence of PMASs on psychological factors and social participation; increased range of and independence in several activities, including eating, drinking, and exercise; variations in funding and time taken for provision; participants' initial reactions; timing of initial use; reasons for nonuse; and suggestions for future development. We concluded that, in this group, PMASs improved confidence, dignity, and the ability to engage in social situations, as well as increased independence in several activities. The majority of negative aspects of use would be amenable to change.
\end{abstract}

Key words: activities of daily living, assistive technology, drinking, Duchenne muscular dystrophy, eating, exercise, feeding, mobile arm supports, neuromuscular conditions, occupational therapy, orthotic devices, qualitative research, rehabilitation, upper limb.

\section{INTRODUCTION}

Neuromuscular conditions (NMCs) are a prominent cause of bilateral upper-limb weakness. In many of these conditions, the proximal musculature is affected first and the distal last, resulting in a relative preservation of hand function while the ability to lift the limb and make use of that function is lost [1]. The combined prevalence of the four commonest NMCs that cause proximal upper-limb weakness-Duchenne, Becker, facioscapulohumeral, and limb girdle muscular dystrophies-was estimated at 21.8/ 100,000 in a recent study of population prevalence in northern England [2] and so represents a notable proportion of the population.

Mobile arm supports (MASs) are a type of assistive technology that enable people with NMCs to make functional use of their hands [3]. They do this by supporting

\footnotetext{
Abbreviations: $\mathrm{ADL}=$ activity of daily living, $\mathrm{MAS}=$ mobile arm support, NMC = neuromuscular condition, OT = occupational therapist, PMAS = powered mobile arm support, PSI = Patient-Specific Index, ULFI = Upper-Limb Functional Index. *Address all correspondence to Margaret Frances Phillips, MD; Division of Rehabilitation Medicine, School of Graduate Entry Medicine and Health, University of Nottingham, Royal Derby Hospital, Uttoxeter Road, Derby, DE22 3DT United Kingdom; 01332-724842; fax: 01332-785681.

Email: margaret.phillips@nottingham.ac.uk http://dx.doi.org/10.1682/JRRD.2012.03.0047
} 
the limb against gravity so the person can use his or her reduced strength to move the limb at the elbow and wrist. Some of the more recent devices have been powered, such as by adjusting the height of the device electronically to allow vertical movement (Neater Arm Support, Neater Solutions Ltd; Buxton, United Kingdom). While these devices have been in use for many years, no qualitative studies have examined user experiences and perspectives of any type of MAS. There are studies using quantitative designs. The largest MAS study was by Haworth et al. in 1978, who investigated a nonpowered MAS that operated using a design based on a plastic bearing and swivel arm [4]. All 124 patients fitted in a 6 yr period were included for the retrospective part of the study, and 77 of them participated in a cross-sectional postal survey. They found that 2 of 18 fitted as outpatients and 40 of 106 fitted as inpatients had stopped using their MASs by 12 mo. Two unselected prospective studies on nonpowered MAS use in those with NMCs include before and after comparisons using report, interview, and medical chart data [4-5]. Yasuda et al. noted that 18 of 29 patients were unsuccessful in their use, although the mean length of use in that group was still $2 \mathrm{yr}$ [5]. The development of the Jaeco/Rancho multilink MAS (JAECO Orthopedic; Hot Springs, Arkansas), which includes use of a power-assist arm-height adjuster and arm retractor, has been described and the device developed with the assistance of a user group [3,6]. A series of four uncontrolled studies evaluating the Armon arm support (Armon, Microgravity Products BV; Rotterdam, the Netherlands) also provides feedback for future prototype development [1,7-9]. The Armon arm is a nonpowered MAS that operates using a statically balanced spring mechanism that can be electronically adjusted according to the load. The first of these studies employed a singlegroup, cross-sectional design in which MAS users were observed completing a series of set tasks or activities of daily living (ADLs) [7], followed by two prospective cohort studies, with possible overlap in participants, reporting on activities completed [1,9]. Finally, a crosssectional study used a validated outcome measure, the Quebec User Evaluation of Satisfaction with Assistive Technology, and a quantitative interview technique, the Individually Prioritized Problem Assessment [8]. This study showed that users could perform several activities felt to be important by them, noted an average level of content with the device, and highlighted some difficulties with the time taken to supply the MAS and practical difficulties in use.

Overall, these studies show that MASs allow users to perform several daily tasks, note specific tasks that can be performed using MASs, demonstrate that type and severity of muscle disease affect use, and provide some insight into difficulties with design and supply through individual comments. The studies also provide information on the technical aspects of design and use. However, they do not include studies of powered MASs (PMASs) or systematically explore the NMC user experience of benefit and problems that would provide us with wider insight into the effects of PMASs on daily life and participation and the range of problems with their use.

Therefore, the aim of this study was to explore the views, experiences, benefits, and difficulties that users of one specific type of PMAS perceive and determine which areas of daily life they are used in. We feel that obtaining the views of users in this research is important because they have the closest experience of using such devices and have experienced their potential and drawbacks. They are also the ones most directly affected by developments in design and provision of PMASs. Such participatory involvement will provide important insights to further the development of PMASs according to user needs and identify recommendations for clinical practice. These recommendations could include the personnel involved in initial assessment, the initial information about PMASs, the process of providing PMASs, the methods of responding to problems, the effect of PMASs on independence and care needs, and the range of activities PMASs could be used for. (See Appendix 1, available online only, for example of participant using PMAS.)

\section{METHODS}

We used a mixed-methods approach, with questionnaires providing demographic and descriptive information about participants and semistructured interviews eliciting in-depth views, thoughts, and feelings regarding the PMAS. The sampling strategy was purposive, based on diagnosis, age, and postcode. We included PMAS users aged $12 \mathrm{yr}$ and over who had an NMC and lived within $200 \mathrm{mi}$ of Derby, United Kingdom. This geographical area includes a large population with a wide range of socioeconomic backgrounds. 
The research team devised the interview schedule (Appendix 2, available online only) after review of the literature. We piloted the interview schedule with a PMAS user, asking him whether he could understand the questions easily and the questions covered the spectrum of PMAS use. We then refined the interview schedule based on his replies and the researchers' own experience of applying the interview schedule. The semistructured interviews followed an iterative process informed by Grounded Theory [10], beginning the interview by raising generative questions to guide the research until "saturation" was achieved and no new ideas were emerging. We used the ABILHAND [11-12] and the Upper-Limb Functional Index (ULFI) [13] to describe upper-limb use and assess the ecological validity of these questionnaires.

As no alternative record of users existed, a company supplying PMASs in the United Kingdom (Neater Solutions Ltd) sent a letter of invitation to the 170 users of Neater arm supports with an NMC living in the study area and then sent a reminder 3 wk later. The letter informed them of the study and asked them to return a form to the research team using an enclosed stamped addressed envelope if they wished to be contacted. We sent respondents an age-specific participant information sheet, a consent form, a demographic form, the ABILHAND, and the ULFI. The participant information sheet consisted of one version for 12-15 yr olds and another for those older than $15 \mathrm{yr}$. The consent form versions were for the parents or guardians in the former age group and for the participant in the latter. We arranged to interview participants at their preferred location or by telephone. At the interview, we completed the informed consent, demographic details, questionnaires, and digitally recorded interview.

We transcribed and analyzed interviews using thematic analysis [14]. Codes were deductively and inductively derived and checked to ensure these harmonized with the context of the interview statement. We grouped codes into clusters and categorized them into themes using NVivo8 (QSR International; Victoria, Australia) [15] and then collectively analyzed and discussed emerging themes. Age, the ABILHAND, and ULFI were analyzed using SPSS version 16 (IBM Corp; Armonk, New York), providing descriptive statistics for these measures. We compared items from quantitative measures with themes from the qualitative research to assess whether a discrepancy existed in the activities identified using the upper-limb scales and using the interview.

\section{RESULTS}

\section{Participant Characteristics}

Twenty-two users (13\% response rate) expressed an interest in the study. Two who were under $12 \mathrm{yr}$ and one who had yet to receive the arm support were excluded. We were unable to contact six, because one provided an incorrect telephone number and five requested information by email and did not reply. Thirteen PMAS users were interviewed: seven at home, one at school, and five by telephone. Nine participants had a parent or guardian present during the interview.

Age ranged from 13 to 69 yr (12 men and boys, mean age $25 \mathrm{yr}$, mode age $14 \mathrm{yr}$; and 1 woman, $69 \mathrm{yr}$ ). Information regarding diagnosis, several aspects of PMAS use, occupation, and use of noninvasive ventilation are given in the Table.

\section{Qualitative Results}

The following themes emerged.

\section{Positive Effect on Psychological Factors}

A great advantage described was the increased independence and the feelings and confidence associated with performing more activities. All participants felt that the ability to eat and drink independently was the main benefit, which had important psychological effects. Participant 3 recalled, "I feel more confident because I can feed myself” and participant 8 reported, "without getting fed, it's more like not embarrassing." This greater independence helped participants be more confident and secure. Participant 1 remarked that knowing his arms were securely fastened by the arm support and that this enabled him to react more quickly to different situations were important confidence-boosting factors. Confidence was also boosted by participants' ability to perform basic intimate tasks such as "hugging a parent" or "shaking hands with people” (Figure 1).

Participants who frequently used their PMAS and their families felt very strongly about them, with comments such as "without it I would have been kind of lost" (participant 1) and "I use it all the time, couldn't go without it really" (participant 5) illustrating the heavy reliance that had developed.

There were negative psychological factors. The PMAS could be seen as a sign of increasing disability: "I felt like 'oh no, this is kind of like another sign I'm getting weaker'” (participant 1). The attitudes of others 
Table.

Demographics of study participants.

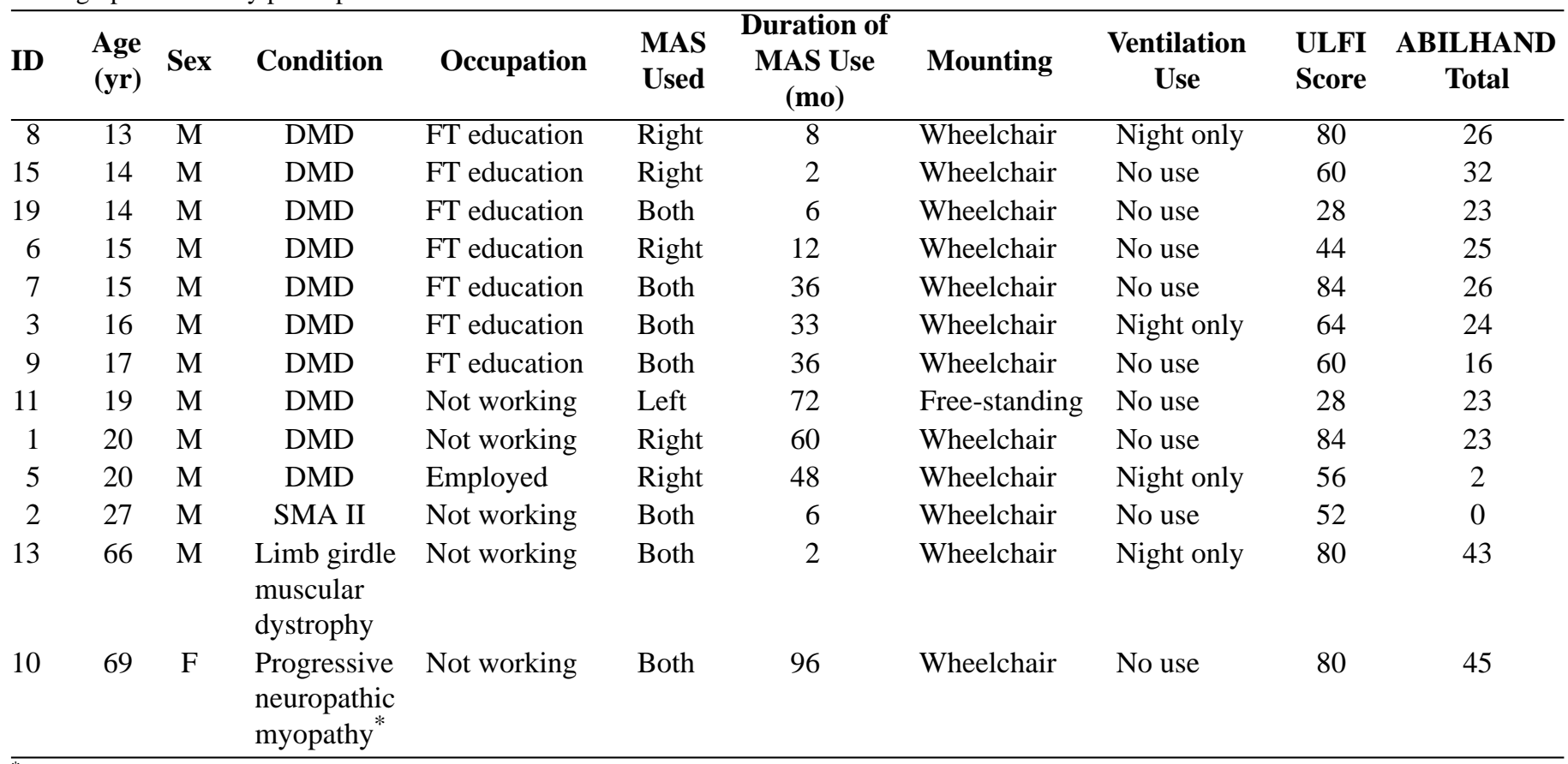

*"Progressive neuropathic myopathy" was participant's own description of her condition.

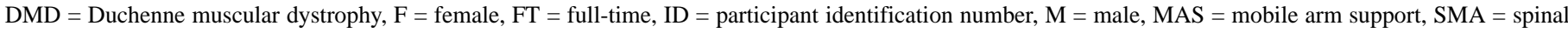
muscular atrophy, ULFI = Upper-Limb Functional Index.

toward them also had a psychological effect, especially when using them in school: "I felt that they stood out, stand out a bit. Thought, but I needed them for eating so I used them at dinnertime" (participant 3). However, this quote also shows negative factors being outweighed by benefits.

\section{Effects on Activity and Participation}

The PMAS allowed several activities to be performed; those described are shown in Figure 2. Comments such as being able to reach further to pick up the television controller, set up a game console, or answer the telephone instead of being reliant on others were common. The ability to perform some tasks more quickly affected other aspects of everyday life: “There's more time in the day to do what I want” (participant 1).

The use of the arm supports also improved posture. Participant 6 recalled that using the arm support for eating helped him to sit upright, as opposed to having his head down to eat, allowing him to talk to others at the table. An effect on aiding digestion was mentioned by several: "Yeah, well because I'm using the arms I'm sat upright rather than leaning on an elbow on the chair, and

\section{Participant 1 Mother:}

I would bring his tea when it's hot and move it out of his way, away from his mouse and keyboard, and when he's ready he would sort of stretch his arm out with the help of the arm support and move his tea closer to him. So in that way he doesn't have to call me, I'll just leave the tea there where he can reach, so that gives him like independence of helping himself. And if it's worth anything, he can hug me back when I hug him; that was the nicest part for me, that he can hug me back. . . Then with the arm support he can actually just hold his arm up and then you can feel it continue, it's the nicest feeling. It sounds silly, but it's the nicest feeling.

\section{Participant 1:}

I do like when I can hug back because I think it's just selfish just taking hugs.

Figure 1.

Illustration of how powered mobile arm support can assist in independence and in enhancing personal relationships. 


\begin{tabular}{|l|}
\hline Maintaining Body Posture \\
Sitting \\
Mobility \\
Using wheelchair by switching it on \\
Opening doors \\
Personal Activities Associated with Reaching \\
Adjusting glasses \\
Reaching \\
Scratching itch \\
Brushing teeth \\
Feeding \\
Preparing food \\
Doing exercises \\
Stretching \\
Socializing \\
Shaking hands \\
Going out to restaurant \\
Leisure Activities \\
Controlling television \\
Using stereo or DVD \\
Using games console \\
Playing piano \\
Drawing \\
Communication and Information Use Through Various Media \\
Writing \\
Picking up telephone \\
Using computer \\
Using keyboard \\
Turning over pages of books and magazines \\
\hline
\end{tabular}

Figure 2.

Aspects of activity and participation that users cited as being assisted by powered mobile arm support. Although displayed by type, some activities could be placed in more than one category.

that does tend to aid digestion because you're not squashing up your gut” (participant 13).

\section{Participants' Initial Reaction to and Use of Their PMAS} and Influencing Factors

Participants varied in their reactions to getting their PMAS, influenced by what they knew about the arm supports and their expectations. Common reactions often focused on the robotic-like appearance of the arm, including participants describing it as "like a robot arm" (participant 6), "they were a bit weird" (participant 7), and "I thought it was a bit strange the way they looked" (participant 9). Mixed emotions occurred, with the excitement of being able to use the arm supports to perform different activities countered by recognition that using them was a sign of further deterioration. Often par- ticipants recalled high expectations of what they would be able to accomplish. Having a demonstration of use of the arm support, either by company promotion or knowing someone else using the arm supports, was described as a further motivational factor in wanting to use them and in maintaining this use. Sometimes they expressed disappointment at not being able to do everything they anticipated because of the lack of muscle strength and mobility: "I thought it would help with all sorts, different kind of stuff, I thought I would be able to pick stuff up and things, and pick bottles of pop up and stuff, but when I got it I wouldn't be able to pick heavy stuff up. But I thought that would do that but obviously it didn't" (participant 8). Training in use varied: "It's trial and error really ... " (mother of participant 8). The mother of participant 11 recounted how, having received the arm support, they were confused about how to set it up and recalibrate it and experienced difficulties with the sling being too small, but that the "the OT [occupational therapist] was quite heavily involved ....” A follow-up visit from the company helped resolve such issues, but highlighted that not all the OTs were experienced in the use and setup of PMASs.

\section{Timing of Obtaining and Learning to Use PMAS and Influence of Severity of Condition on Extent and Range of Use}

The timing of prescription of a PMAS in relation to disease severity was important, especially in terms of having enough remaining strength to get used to them: "So yeah, also the timing of getting the arm support was I think perfect, ... . at that moment where I still had enough mobility to kind of do a few things myself, and kind of get used to the arm support before it's kind of too difficult to get used to something like that” (participant 1).

Participants who had to wait a long time to get their PMAS had become weaker while waiting, which may have influenced use. Others experienced initial discomfort and aching because they were using muscles not used for some time, although this wore off. Participant 11 remarked, "first of all I couldn't get on with it, but I had to just keep trying and I'm brilliant at it now.”

The specific muscle groups affected influenced the extent to which the PMAS could be used. For example, one participant with severe limb girdle muscular dystrophy (participant 13) was limited in upper-limb abduction “because they’re hinged and they, you can’t anchor them, 
they're moving in and out and I don't think I have the power, muscle power to move them in and out.”

\section{Reasons for Not Wanting to Use PMAS}

Younger participants were more reluctant to use the arm supports. Four used them primarily for feeding and physiotherapy, but disliked using their arm supports in the classroom. One participant (participant 7) had recently stopped using his arm supports in school. When questioned further about this he recalled, "I didn't want to bother at school” since they were "a bit hard [for the support workers] to put them on." Yet despite not using the arm supports at school, participant 7 regularly used them at home for eating, playing console games, and exercising; he described that, when using them, he enjoyed eating more and had better posture. Other problems experienced with use at school were difficulty transporting them, time needed to set them up, and feeling self conscious: "I was just a bit embarrassed because it stuck out a bit, and it's a bit, I just weren't very happy using it” (participant 11).

When asked when they would purposefully not use their PMAS, most participants cited when traveling in a motor vehicle, whether as passenger or driver. This was because their arm slipped out of the sling. The increase in wheelchair width due to the PMAS led to difficulty moving around the house, with many homes having narrow doorways. One participant recalled, "when we had a party at Christmas I didn't use them because of lack of space” (participant 10).

Occupational Therapists Raising Awareness and Advocating for Funding

An OT was most often the key individual who raised awareness about the arm supports and advocated for their use, but OT experience, involvement, and knowledge varied. Some participants recalled that their OT recommended use because of experience with other patients using PMASs.

\section{Variation Across Local Authorities in Funding and Time Taken to Provide PMAS}

The wait to get PMAS varied from months to almost $2 \mathrm{yr}$, often because of funding. Some participants obtained funding from their local authority, but this was a struggle, often requiring strong persuasion by their OT. Participant 3 related how they gave up waiting for funding to come through and purchased the arm supports privately: "It would have taken too long so we just decided to buy them" (mother of participant 3). Three participants obtained funding quickly for their PMAS through Tesco's funding in 2009 when the Muscular Dystrophy Campaign was its charity of the year.

\section{Users' Suggestions for Further Development}

Some participants and their families felt they could have received better support following supply of the PMAS and that they could reach further than the arm support would allow them to, perceiving this as a limitation: “. . . one of the disadvantages I find is kind of the reach it has, that's as far as I can reach but I can feel my body's got more stretch in me to be able to reach further" (participant 1).

Because of the design of the current PMAS, both arms move at the same time. This design received mixed reviews from participants, with comments such as "I couldn't get the angle right, so I couldn't comfortably use them both at the same time” (participant 2) and “. . . you don't always want two arms up in the air if you're trying to reach for something off a shelf” (participant 10). Some participants found that the sling for the arm supports provided insufficient wrist support, and several described their arm falling out of the sling when the supports were used to raise their arms: "Sometimes, when I feed myself my arm slips out of the sleeve because of the angle ... So I suppose maybe having a sling that would hold the arm better without having to strap it in" (participant 2). Common issues were that the switch broke and the wires were caught easily: "A better switch. They give you a switch to start off with, and they always break. We get them ourselves now. I'm on about the tenth . . . because the wires just snap inside" (participant 3). Further suggestions included a bag to carry them and a fastener to ensure that the arm supports could be tied behind the wheelchair when not in use, allowing easier access through doors or around a corner.

\section{Quantitative Results}

Participants achieved a median of 60 percent, range 28-84 percent, for the ULFI. A median score of 80 percent, range 56-80 percent, was obtained for the PatientSpecific Index (PSI) of the ULFI, indicating how well users performed five activities important to them. The PSI described a wide range of activities that corresponded well to those identified in the interviews. Participants noted problems interpreting the measure because it asked respondents to identify activities that their upper-limb 
problem (as opposed to condition) caused difficulties with. The median value for the ABILHAND was 7, range 0-24 (maximum attainable score 46), with many activities not attempted. Activities were identified that were not during the interviews, such as threading a needle and wrapping gifts, but items were also identified during interviews that were not included in the ABILHAND, such as adjusting glasses and scratching an itch.

\section{DISCUSSION}

This study provides important insights into the use of PMAS, giving us further information on benefits and difficulties in use from the perspective of users with NMC. It shows that PMASs increase independence in a wide range of activities, affecting not just physical independence but also psychological aspects such as confidence and ability to engage in social situations. Negative aspects of use were identified, many of which are amenable to change, such as knowledge of capabilities of the PMAS before supply, training, design, and waiting times, but also some that are more difficult to address, such as the attitude of others, funding, and the PMAS being a sign of increasing disability.

Use of a PMAS leads to independence in several activities, which could reduce care costs, most notably for feeding and educational support, and independence in other activities that may improve quality of life more than care costs. However, even these latter aspects may reduce carer strain, which could have an indirect effect on care costs and health service utilization. The effects on various ADLs, such as eating, drinking, using a keyboard, and adjusting spectacles, have been noted in previous studies $[4,6,16-18]$. The reported effects on posture and gastrointestinal symptoms are new observations that could have secondary implications for respiratory function and comfort. Positive effects here could lead to a reduction in healthcare costs and the effect on posture needs to be considered in prescription of wheelchair seating, both of which could be tested by further study.

Timing of provision and the amount of training needed were important related aspects identified, also noted in the study of the Armon arm support by Lund et al. [8]. To maximize benefit from use of PMASs, learning to use them while the user still has enough strength to easily manipulate them may both improve initial adjustment and potentially assist in maintaining a higher degree of muscle strength for longer $[3,8]$. This is because the upper limbs are not used at all once antigravity strength is lost unless they can be assisted by an external device such as the PMAS and so are liable to additional weakness due to disuse atrophy [19]. The early introduction of equipment for independent living is recommended to facilitate optimal use and increased benefit for carers [20]. Further prospective research could explore this and determine the optimum time of prescription of a PMAS that will minimize strength lost through disuse and aid in retention of key upper-limb activities. Criteria for use originate from recommendations made by Wilson et al. [21] and have been assessed in NMC [5] and spinal cord injury [18] populations, but these note the strength that is required for successful, rather than optimal, use. We also noted that the initial learning phase is compromised by differences in teaching physical and technical aspects of use, showing similarities with the study by Lund et al. [8]. This could be improved by suppliers and therapists working together by setting up a rehabilitation program driven by patient-negotiated goals that included education in use of the device, addressing technical issues and with follow-up support to readjust and answer queries. This would be true rehabilitation of upper-limb function rather than simple supply of equipment. The use of patient-negotiated goals within the context of a rehabilitation program is well known to provide the motivation to use equipment in a functional way [22].

Despite the ability to perform tasks more independently and quickly, school-aged participants showed a notable absence of motivation for use of the PMAS in schools. The school-aged participants expressed a reluctance to use their PMAS in the classroom despite regular use at home and their acknowledgment of perceived benefits of use. This is in contrast to the successful use of the Arlyn arm robotic station noted by Eberhardt et al. [23], who showed that students could independently carry out science experiments and art projects in the classroom. However, that study only included five participants and examined specific tasks rather than use over a period of time. It is well known that feelings of not wanting to appear different from peers may hamper use of assistive technologies in the classroom [24]. Our study highlights a need to investigate further the inconsistency of PMAS nonuse in schools and explore how school-aged PMAS users could be better supported.

Several other aspects were identified that were important in successful use of the PMAS. Successful 
communication about its capabilities when first proposed is important. Problems with reliability of the switch and wires had a major effect on acceptance for some users, as did the ability of the PMAS to provide sufficient wrist support. There were individual preferences in whether to use one PMAS or two. The additional width as a result of the PMAS was identified as a determining factor for where and when the PMAS could be used. It highlights how the extra width added to the wheelchair needs to be considered at the time of wheelchair and PMAS prescription, particularly in relation to the environment in which the user will be using the wheelchair/PMAS combination [25]. Such understanding and insight into the experience of those who use the PMAS promote user-driven development of the PMAS, rather than the tendency for technology-driven assistive robotics [26]. The aspects of performance and consideration of the user's opinion when first proposed have been recognized as important in ensuring continued use of assistive technology for many years [27].

The issue of funding was apparent, with problems in obtaining the PMAS and delays in provision due to the application process. One positive note was the difference that charitable funding had made for some, although this was the result of a specific initiative that increased the ability of a specific charity to fund equipment that year. This issue has been raised previously in the general context of funding for electronic assistive technology [28], where it was noted that a large amount of time was spent in applying for such grants. Funding may be provided to purchase equipment, as practiced by the Muscular Dystrophy Campaign in the United Kingdom, or to loan items from an equipment bank, such as that maintained by the Muscular Dystrophy Association in the United States.

The ULFI and ABILHAND identified a range of activities that were influenced by the PMAS. However, possibly due to item reduction during questionnaire development, the interviews identified a greater range and depth of activities than the ULFI and ABILHAND, meaning that the content validity of these measures was limited in this group of participants. The participants identified problems in interpreting both measures, which could only be rectified by adding to the instructions given.

There were several limitations to the study. The response rate was low, raising the concern that only successful users may have replied. However, we received a number of negative comments and some users were using their PMASs very little, suggesting that participants were not just those with positive experiences and higher degrees of motivation. We emphasized in the invitation letter that participants did not have to be frequent users, but we would not have identified those who trialed the PMAS and decided not to have them, so this study should be interpreted as reflecting the views of current users of PMASs. The majority of our participants were young men and boys with Duchenne muscular dystrophy, so there may have been aspects not identified that were important to older users with other NMCs, especially women. We did not record race/ethnicity or socioeconomic status, although there were participants from a variety of racial/ethnic and socioeconomic backgrounds in the study. If these were not fully represented, we may have missed issues that were specific to some cultures or socioeconomic backgrounds. Family and carer support may be important, but we did not explore this aspect. Finally, the PMASs studied were those produced by one company to limit variability in the devices being studied and assist in focusing the themes, but this may have resulted in some aspects being less transferable to other types of PMASs.

\section{CONCLUSIONS}

This study emphasizes that use of PMASs by adults and teenagers with NMCs can greatly improve their independence, confidence, and ability to engage in social situations. It has highlighted aspects of PMAS use that were not previously identified, such as a beneficial effect on posture and gastrointestinal symptoms, negative and positive aspects of their use in schools, a more detailed and comprehensive list of disadvantages, areas where their use may reduce care costs, and areas where they may improve quality of life. The identified problems with funding may be an issue specific to the United Kingdom, but would have relevance to other health and social care systems. In addition, this study has confirmed aspects identified previously, such as problems with timing of provision and debate over whether PMASs may be used to reduce the rate of deterioration of muscle strength. Finally, the study identified that two current activitybased upper-limb measures did not have good content validity when used in this context, which is important as regards choosing measures for future studies. 


\section{ACKNOWLEDGMENTS}

\author{
Author Contributions: \\ Study conception and design: A. Kumar, M. F. Phillips. \\ Acquisition of data: A. Kumar, M. F. Phillips. \\ Analysis and interpretation of data: A. Kumar, M. F. Phillips. \\ Drafting of article: A. Kumar. \\ Revision of article: A. Kumar, M. F. Phillips. \\ Approval of final version: A. Kumar, M. F. Phillips.
}

Financial Disclosures: The authors have declared that no competing interests exist. Ipsen Pharmaceuticals had no role in the study design; collection, analysis, and interpretation of data; writing of the report; or decision to submit the report for publication.

Funding/Support: This material was based on work supported in part by an educational grant from Ipsen Pharmaceuticals.

Additional Contributions: Sincere thanks goes to all the MAS users and their families who contributed to this study by sharing their experiences and views. Arun Kumar is now with the Division of Primary Care at the University of Nottingham.

Institutional Review: The University of Nottingham Ethics Committee approved this study. All participants signed an informed consent form.

Participant Follow-Up: The authors do not plan to inform participants of the publication of this study because contact information is unavailable. However, they will inform the Muscular Dystrophy Campaign of the publication of the study in case they wish to put it on their Web site.

\section{REFERENCES}

1. Herder JL, Vrijlandt N, Antonides T, Cloosterman M, Mastenbroek PL. Principle and design of a mobile arm support for people with muscular weakness. J Rehabil Res Dev. 2006;43(5):591-604.

2. Norwood FL, Harling C, Chinnery PF, Eagle ME, Bushby K, Straub V. Prevalence of neuromuscular disease, including CMD in Northern England. Brain. 2009;132:3175-86. [PMID:19767415] http://dx.doi.org/10.1093/brain/awp236

3. Landsberger S, Leung P, Vargas V, Shaperman J, Baumgarten J, Yasuda YL, Sumi E, McNeal D, Waters R. Mobile arm supports: history, application, and work in progress. Top Spinal Cord Inj Rehabil. 2005;2(11):74-94.

4. Haworth R, Dunscombe S, Nichols PJ. Mobile arm supports: an evaluation. Rheumatol Rehabil. 1978;17(4):240-44. [PMID:153577] http://dx.doi.org/10.1093/rheumatology/17.4.240

5. Yasuda YL, Bowman K, Hsu JD. Mobile arm supports: criteria for successful use in muscle disease patients. Arch Phys Med Rehabil. 1986;67(4):253-56. [PMID:3964060]

6. Landsberger S, Shaperman J, Vargas V, Meadows E, Mitani M, McNeil DR. Mobile arm supports for children, an update. ACPOC News. 1999;5(2):5-7.

7. Herder JL. Development of a statically balanced arm support: ARMON. IEEE 9th International Conference on
Rehabilitation Robotics; 2005 Jun 28-Jul 1; Chicago, IL. 2005. p. 281-6.

8. Lund K, Brandt R, Gelderblom GJ, Herder JL, editors. A user-centered evaluation study of a mobile arm support. IEEE 11th International Conference on Rehabilitation Robotics; 2009 Jun 23-26; Kyoto, Japan.

9. Mastenbroek B, de Haan E, van den Berg M, Herder JL, editors. Development of a mobile arm support (Armon); design evolution and preliminary user experience. IEEE 10th International Conference on Rehabilitation Robotics; 2007 Jun 13-15; Noordwijk, the Netherlands.

10. Martin PY, Turner BA. Grounded theory and organizational research. J Appl Behav Sci. 1986;22(2):141-57. http://dx.doi.org/10.1177/002188638602200207

11. Penta M, Tesio L, Arnould C, Zancan A, Thonnard J-L. The ABILHAND questionnaire as a measure of manual ability in chronic stroke patients: Rasch-based validation and relationship to upper limb impairment. Stroke. 2001; 32(7):1627-34. [PMID:11441211]

12. Vandervelde L, Van den Bergh PY, Penta M, Thonnard J-L. Validation of the ABILHAND questionnaire to measure manual ability in children and adults with neuromuscular disorders. J Neurol Neurosurg Psychiatry. 2010;81(5):506-12. [PMID:19726413] http://dx.doi.org/10.1136/jnnp.2009.177055

13. Gabel CP, Michener LA, Burkett B, Neller A. The Upper Limb Functional Index: development and determination of reliability, validity, and responsiveness. J Hand Ther. 2006; 19(3):328-48. [PMID:16861132] http://dx.doi.org/10.1197/j.jht.2006.04.001

14. Braun V, Clarke V. Using thematic analysis in psychology. Qual Res Psychol. 2006;3(2):77-101. http://dx.doi.org/10.1191/1478088706qp063oa

15. QSR International Pty Ltd. NVivo qualitative data analysis software version 8. Doncaster (Australia): QSR International Pty Ltd; 2008.

16. Herder JL, Vrijlandt N, Antonides T, Cloosterman M, Mastenbroek PL. Principle and design of a mobile arm support for people with muscular weakness. J Rehabil Res Dev. 2006;43(5):591-604. [PMID:17123201] http://dx.doi.org/10.1682/JRRD.2006.05.0044

17. Lund K, Brandt R, Gelderblom G-J, Herder JL, editors. A user-centred evaluation of a mobile arm support. IEEE 11th International Conference on Rehabilitation Robotics; 2009 Jun 23-26; Kyoto, Japan.

18. Atkins MS, Baumgarten JM, Yasuda YL, Adkins R, Waters RL, Leung P, Requejo P. Mobile arm supports: evidencebased benefits and criteria for use. J Spinal Cord Med. 2008;31(4):388-93. [PMID:18959356]

19. Jansen M, de Groot IJ, van Alfen N, Geurts AC. Physical training in boys with Duchenne Muscular Dystrophy: the 
JRRD, Volume 50, Number 1, 2013

protocol of the No Use is Disuse study. BMC Pediatr. 2010;10(1):55. [PMID:20691042]

20. Paul SN, Frank AO, Hanspal RS, Groves R. Exploring environmental control unit use in the age group 10-20 years. Int J Ther Rehabil. 2006;13:511-16.

21. Wilson DJ, McKenzie MW, Barber LM, Watson KL. Spinal cord injury: a treatment guide for occupational therapists. Thorofare (NJ): SLACK Inc; 1984.

22. Wade DT. Goal setting in rehabilitation: an overview of what, why and how. Clin Rehabil. 2009;23(4):291-95. [PMID:19293289] http://dx.doi.org/10.1177/0269215509103551

23. Eberhardt SP, Osborne J, Rahman T. Classroom evaluation of the Arlyn Arm robotic workstation. Assist Technol. 2000;12(2):132-43. [PMID: 11508403]

24. McIntosh M. Resistance is futile: assistive technology and students with learning disabilities. Henderson (MN): Closing the Gap Solutions; 2010.

25. Evans S, Neophytou C, de Souza L, Frank AO. Young people's experiences using electric powered indoor - outdoor wheelchairs (EPIOCs): potential for enhancing users' development? Disabil Rehabil. 2007;29(16):1281-94. [PMID:17654003] http://dx.doi.org/10.1080/09638280600964406

26. Sixsmith A, Sixsmith J. Smart care technologies: meeting whose needs? J Telemed Telecare. 2000;6 Suppl 1:S190-2.
27. Phillips B, Zhao H. Predictors of assistive technology abandonment. Assist Technol. 1993;5(1):36-45.

[PMID:10171664]

http://dx.doi.org/10.1080/10400435.1993.10132205

28. Cowan D, Turner-Smith A. The funding agencies' perspective on the provision of electronic assistive technology: equipping for life? Br J Occup Ther. 1999;62(2):75-79.

Submitted for publication March 15, 2012. Accepted May 17, 2012.

This article and any supplementary material should be cited as follows:

Kumar A, Phillips MF. Use of powered mobile arm supports by people with neuromuscular conditions. J Rehabil Res Dev. 2013;50(1):61-70.

http://dx.doi.org/10.1682/JRRD.2012.03.0047

ResearcherID: Margaret Frances Phillips, MD: F-38132011

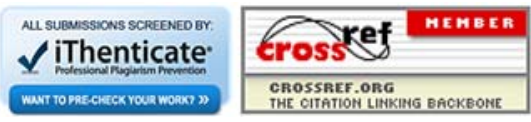

